\title{
Old Arsenal to Combat New Enemy: Repurposing of Commercially available FDA Approved drugs against Main Protease of SARS-CoV2.
}

\section{Gagandeep Singh $^{1 *}$, Vishal Srivastava1, Ritpratik Mishra², Gaurav Goel ${ }^{2}$, Tapan K.}

Chaudhuri $^{1}$

${ }^{1}$ Kusuma School of Biological Sciences, and ${ }^{2}$ Department of Chemical Engineering, Indian Institute of Technology Delhi, New Delhi (India)-110016.

*Corresponding Author

Communication Address:

Kusuma School of Biological Sciences, Indian Institute of Technology Delhi Hauz Khas, New Delhi, India-110016

Phone: +91-8178163366

Fax: +91-11-2659-7530

Email: gagan.sk.1994@gmail.com

\section{Author's Email IDs:}

Gagandeep Singh (Gagandeep.singh.khurana@bioschool.iitd.ac.in)

Vishal Srivastava (vsri.fzd@gmail.com)

Ritpratik Mishra (Ritpratikmishra@gmail.com)

Gaurav Goel (goelg@ chemical.iitd.ac.in)

Tapan K. Chaudhuri (tkchaudhuri@bioschool.iitd.ac.in) 


\begin{abstract}
In lack of vaccination and therapeutic drugs, the ongoing COVID-19 pandemic affected millions of people, causing 1,018,957 deaths worldwide (World health organization; $1^{\text {st }}$ October 2020). The conventional drug design pipeline for effective and safer drug development is a costly and time-intensive affair. It takes around ten years in general from identifying a clinical candidate to get the approvals for actual applications. An effective way to cut short drug design pipeline in such emergency cases could be the repurposing of already approved drugs against novel targets. Here in this work, we explored the structure-based drug screening approach to find potential inhibitors of SARS-CoV2 main protease $\left(\mathrm{M}^{\mathrm{pro}}\right)$ from the library of already FDA approved commercially available drugs. The site-specific and blind docking studies, in combination, suggest three potential inhibitors of $\mathrm{M}^{\text {pro }}$, Ergotamine (ZINC000052955754), Nilotinib (ZINC000006716957) and Naldemedine (ZINC000100378061). Molecular dynamics (MD) simulations and binding free energy calculations using the MMPBSA method further reinforced the efficiency of the screened $\mathbf{M}^{\text {pro }}$ inhibitor candidates. The work yields enough evidence to conduct rigorous experimental validation of these drugs before utilizing them for the therapeutic management of SARS-CoV2 infection.
\end{abstract}

\title{
Graphical Abstract
}

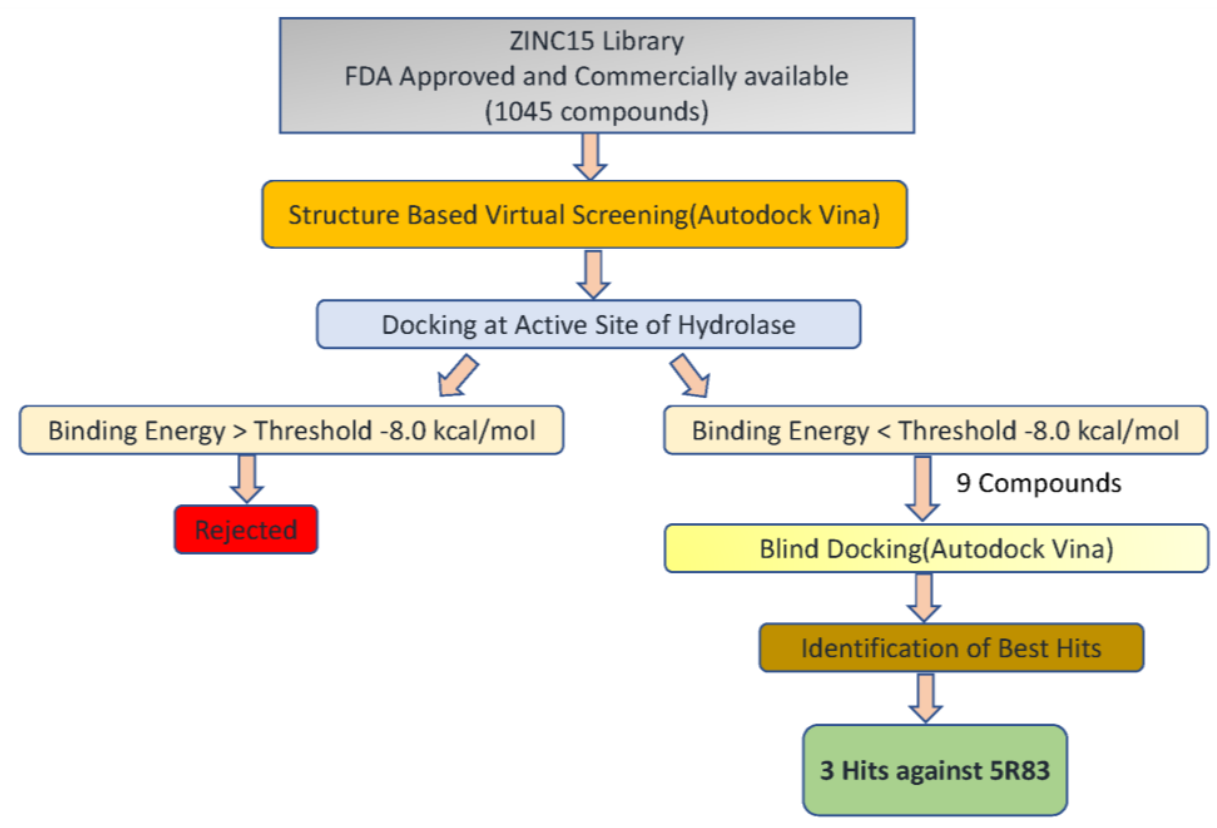




\section{Introduction}

As early as 31st December 2019, a cluster of pneumonia of unknown origin has been reported in Wuhan, Hubei Province, Republic of China. Later the causal organism is reported as a newly mutated strain of SARS Coronavirus (SARS-CoV) having higher pathogenicity and termed as SARS-CoV2 (Severe Acute Respiratory Syndrome CoronaVirus-2) causing the CoronaVirus Disease (COVID-19) ${ }^{1}$. Among all the seven known coronaviruses, SARS-CoV2 is one of the three highly pathogenic human coronaviruses, including the MERS-CoV and SARS CoV. Although SARS-CoV2 exceeds the transmission rate from previously reported outbreaks of human coronaviruses, the virus has a much lower case fatality rate (CFR), i.e., 2-5\% 34.4\% and $10 \%$ reported in the case of MERS-CoV and SARS-CoV respectively ${ }^{2-4}$. The worldwide spread of the disease prompted the World Health Organization (WHO) to declare it a pandemic of a significant threat to public health, which needs to be addressed adequately by prevention, and therapeutics. As per WHO COVID-19 Dashboard, there were 31,798,308 confirmed cases of COVID-19 worldwide resulting in 973,653 deaths on 25th september $2020^{5}$. Clinical manifestation of SARS-CoV2 infection ranges in between being symptomatic to experience severe respiratory failure ${ }^{6}$. Early symptoms of the disease comprise anything or the combination of low-grade fever, fatigue, dry cough, myalgia, dyspnea, lack of taste, and smell ${ }^{7}$. Pneumonia is found to be associated with most of the SARS-CoV2 infections, but cases of having pleuritic chest pain also been reported ${ }^{7}$. Based on the severity of the symptoms, infected patients could be classified into mild, severe, and critical types ${ }^{8}$. However, most infections are self-limiting in nature, leaving approximately $15 \%$ cases to reach a severe level, which requires hospitalization and oxygen supplementation. Based on the reports, an additional 5\% of such patients become critically ill, showing hypoxemia, acute respiratory distress, and multiple organ failure leading to death in the absence of necessary ventilator support for several weeks ${ }^{7,9-13}$.

Similar to other coronaviruses, SARS-CoV-2 is an enveloped spherical viral particles consisting of a 29903 bp (Accession no. NC_045512) extended positive-sense single-stranded RNA (ssRNA (+)) as genetic material. The genomic features of the SARS-CoV2 are wellconserved, showing $79.5 \%$ sequence similarity with early reported SARS-CoV. Despite having high sequence similarity, genetic modifications are highly impactful, resulting in resistance towards those drugs which were previously found effective against SARS-CoV ${ }^{14}$. Virus assembly consists of several protein molecules, viz- spike(S), envelope (E), membrane (M), nucleocapsid (N), major protease (Mpro), replicase-transcriptase, etc. Several of these proteins could serve as essential targets for therapeutic design and development, considering their crucial role in the viral life cycle ${ }^{15-17}$. The spike (S) protein of SARS-CoV2 is a trimeric class I viral fusion glycoprotein, which assists in an initial attachment to the angiotensin-converting enzyme II (ACE2) is known to allow fusion with the host cells such as alveolar epithelial cells $^{8,18,19}$. The protein is an essential target for vaccine and inhibitor drug design ${ }^{3,4}$.

Chymotrypsin, like protease (3CLpro) or SARS-CoV2 major protease (Mpro), is also an attractive target for drug design and development due to high sequence conservation and its 
critical role viral pathogenesis ${ }^{20}$. The protease process the viral polyprotein $1 \mathrm{a} / 1 \mathrm{ab}$, into mature non-structural proteins (mNSPs), which play an essential role in blocking host immune response $^{21}$. However, the primary function of SARS-CoV-2 Mpro is the regulation of replication and transcription machinery of SARS-CoV2 by controlling the synthesis of nonstructural proteins (NSPs) required for replicase-transcriptase assembly ${ }^{22}$. The protein also shows a high sequence similarity, i.e., 96\% with SARS-CoV major protease (Mpro). It makes Mpro an ideal target for structure-based drug design and development. SARS-CoV2 Mpro is a homodimer of 306 amino acid long polypeptide chains. Crystal structure analysis suggests amino acid residues His41, Phe140, Leu141, Asn142, Gly143, Cys145, His163, Met165, Glu166, Leu167, Pro168, Phe185, Asp187, Gln189, Thr190, Ala191, and Gln192 define the substrate-binding pocket of protease ${ }^{20,22,23}$. At the same time, the His41 and Cys145 served as a part of the catalytic dyad. These residues could be regarded as prime targets during structurebased drug development to measure their interaction with potential ligand molecules to obtain SARS-CoV2 Mpro inhibitors ${ }^{24-26 .}$.

The repurposing of approved drugs provides an alternative approach to develop safe and effective therapeutics in case of rapidly emerging diseases and sudden outbreaks, as in the case of COVID-19 ${ }^{27}$. While the conventional drug development pipeline is time-intensive and costs millions, repurposing already available drug molecules could result in the timely development of effective and safe drugs for clinical testing and applications ${ }^{15,17,24}$. In this work, we performed the structure-based virtual screening of the FDA-approved commercially available drugs to find high-affinity inhibitors of SARS-CoV-2 Mpro. First, we estimated the drugbinding affinities with SARS-CoV-2 Mpro and then conducted the interaction analysis to find better hits. Finally, based on the interaction analysis and drug properties, we identified two drugs, viz- Ergotamine and Naldemedine showing the appreciable binding affinity and specific interactions toward the SARS-CoV2 Mpro active site.

\section{Materials and Methods}

\section{1- Protein and Ligand Preparation}

SARS-CoV2 crystal structure having PDB ID-5R83 has been studied extensively along with other crystal structures such as 5R7Y, 5R7Z, 5R80, 5R81, 5R84, 5RE4, 5RE9, 5REB, 5RF1, $5 \mathrm{EFQ}, 6 \mathrm{LU} 7$, and $6 \mathrm{~W} 63$ to identify the critical residues involved in $\mathrm{M}^{\text {pro }}$-inhibitor interaction and understand the mechanism of inhibition. For molecular docking-based virtual screening, atomic coordinates of SARS-CoV2 $\mathrm{M}^{\text {pro }}$ were obtained from the PDB ID 5R83. The crystal structure contained 304 amino acid residue long single chain of SARS-CoV2 $\mathrm{M}^{\text {pro }}$ in complex with hydrolase inhibitor Z44592329 at $1.58 \AA$ A. Water molecules and hydrolase inhibitor were removed, and the geometry of the protein was cleaned through the "Prepare Protein" module of Biovia Discovery Studio 4.5(DS4.5).

SDF file representing the library of 1045 FDA approved and commercially available drugs were downloaded from the ZINC15 database (https://zinc15.docking.org/). Energy minimization of all the 1045 ligand molecules was performed in vacuo, using 500 steps of conjugate gradients optimization algorithm with universal forcefield updated at one step 
interval. The energy minimization process was stopped at an energy difference of $0.1 \mathrm{kcal}^{\mathrm{mol}}{ }^{-}$ ${ }^{1}$. Further, the energy minimized ligand molecules were converted to Autodock ligands (.pdbqt) format using PyRx 0.8 (https://pyrx.sourceforge.io/).

Table-1: Grid box size and coordinates used for docking in Autodock Vina.

\begin{tabular}{lll}
\hline & $\begin{array}{l}\text { Active site Docking } \\
\text { On 5R83 }\end{array}$ & $\begin{array}{l}\text { Blind-Docking } \\
\text { On 5R83 }\end{array}$ \\
\hline Center(̊) & & \\
X & -29.9394 & -39.995 \\
Y & -39.3462 & -26.077 \\
Z & 14.5804 & 14.7781 \\
Box Dimensions $(\AA)$ & & \\
X & 41.6726 & $\mathbf{5 7 . 6 8 2 1}$ \\
Y & 33.7555 & 64.0011 \\
$Z$ & 49.6611 & 48.8962
\end{tabular}

\section{2- Structure-based virtual screening}

PyRx 0.8, along with AutoDock Vina v.1.1 ${ }^{28}$, was used for structure-based docking studies of Mpro with FDA approved ligand molecules. The software was selected owing to its rapid yet efficient search capability to find accurate and active conformations ${ }^{29}$. While the SARS-CoV2 Mpro protein molecule was considered as a rigid structure, ligand drug molecules were considered flexible during the docking process. Molecular docking at the Active site of $\mathbf{M}^{\text {pro }}$ was performed using AutoDock Vina (V1.1) for all the ligand drug molecules with a grid size of $41.67,33.75$, and $49.66 \AA$, centralized at $-29.93,-39.34$, and $14.58 \AA$ for $\mathrm{X}, \mathrm{Y}$, and Z coordinates as mentioned in the table-1. The RMSD of $2 \AA$ was found in the overlapped structure of the native inhibitor in the crystal form and the virtually docked form, as shown in Figure 1. The assessed binding energy score of the native hydrolase inhibitor (Z44592329) in the crystal structure (PDB ID: 5R83) was found $-6.0 \mathrm{kcal}^{\mathrm{mol}}{ }^{-1}$. The threshold value for binding energy was set to $-8.0 \mathrm{kcal}^{\mathrm{mol}} \mathrm{l}^{-1}$ to narrow down the search space to obtain only the highaffinity ligand molecules to SARS-CoV2 $\mathrm{M}^{\text {pro }}$ as a target. A total of 9 out of 1045 compounds showed binding energy $\left(\mathrm{DG}^{\circ}\right)$ less than $-8.0 \mathrm{kcal} . \mathrm{mol}^{-1}$ showing the high affinity of these ligand molecules towards $\mathrm{M}^{\text {pro }}$ active site (Table-2).

\section{3- Blind docking studies}

Another round of structurally blind docking was performed with initially screened nine FDA approved drug ligand molecules using AutoDock Vina. The box dimensions for structurally blind docking were 57.6864 .00 and $48.89 \AA$ while the $\mathrm{M}^{\text {pro }}$ was centralized at $-29.93,-39.34$, and $14.58 \AA$ for $\mathrm{X}, \mathrm{Y}$, and $\mathrm{Z}$ coordinates during the docking (Table-1). The grid spacing was set to $1.00 \AA$. The blind docking was performed to cover the entire protein structure under the grid search space to check whether the primary screened molecules have an affinity to bind to the enzyme other than the active site. Six out of the previously screened nine compounds 
showed comparable or high affinity to the sites other than the target's active site. Remaining three molecules, Ergotamine, Naldemedine, and Nilotinib, had shown a high affinity to the active site having minimal or no affinity to other sites. The docked poses of which are shown in Figure 2, and selected further for detailed analysis.

\section{4- Inhibition constant calculation}

The inhibition constant selected ligand molecules has been calculated from the obtained $\Delta G$ parameter from molecular dynamics simulations and putting the values in the following formula-

$$
\begin{aligned}
\Delta G & =\operatorname{RT}\left(\operatorname{Ln} K_{\mathrm{i}}\right) \ldots .(1) \\
K_{\mathrm{i}} & =\mathrm{e}(\Delta G / \mathrm{RT}) \ldots(2)
\end{aligned}
$$

The value is represented in terms of the negative decimal logarithm of inhibition constant $\left(\mathrm{p} K_{\mathrm{i}}\right)$

$$
\mathrm{pKi}(\mu \mathrm{M})=-\log \left(K_{\mathrm{i}}\right) \times 10-6 \ldots(3)
$$

Where $\Delta G$ is binding affinity (kcal.mol $\left.{ }^{-1}\right), R$ (gas constant) is 1.98 cal. (mol.K) $)^{-1}$, and $T$ (room temperature) is 298.15 Kelvin.

\section{5- Molecular dynamics (MD) simulation studies}

Molecular dynamics (MD) simulation studies were conducted using $\mathrm{LiGRO}^{30}$, a GUI based MD simulation software with GROMACS 5.1.5 package $^{31}$ to evaluate the metastability of the native inhibitor, and screened drug ligands with SARS-CoV2 $\mathrm{M}^{\text {pro }}$. Transferable intermolecular potential water molecules (Tip3p) model for solvation along with Amber FF99sb forcefield was applied. The complex was placed inside a dodecahedron box $(60 \times 60 \times 90 \AA)$ containing 35,897 water molecules, such that the minimum distance between any atom of the proteinligand complex with the box wall was $2 \mathrm{~nm}$. The parameters of ligands were calculated using General Amber Force Field (GAFF) and AnteChamber PYthon Parser interfacE (ACPYPE) ${ }^{32}$ module in LiGRO. The protein-ligand complex with an overall -3 charge was neutralized with $0.15 \mathrm{M}$ concentration of $107 \mathrm{Na}+$ and $103 \mathrm{Cl}$ - ions. The equilibration steps under NVT and NPT ensemble were run for $1 \mathrm{~ns}$ each in LiGRO, and the MD production run for each complex and free $\mathrm{M}^{\text {pro }}$ was run under NPT ensemble at 1.01 bars at $310.15 \mathrm{~K}$ for $100 \mathrm{~ns}$ each at High performance cluster (HPC) facility at IIT Delhi. The particle mesh Ewald (PME) method and LINCS algorithm were applied to correct the electrostatic interactions and constrain the bonds with hydrogen atoms. The time step was $2 \mathrm{fs}$, and the frames were stored after every 500 steps. The trajectories and images were visualized through $\mathrm{VMD}^{33}$ and Chimera Software ${ }^{34}$.

The obtained data were analysed using GROMACS tools (rmsd, rmsf, and energy) to examine the overall stability of the protein-ligand system, fluctuations in the local residues, and structure over the complete simulation trajectory. In this study, MM-PBSA calculations were done using the g_MMPBSA ${ }^{35}$ software, and the $\Delta \mathrm{G}$ was calculated by equation (1), 


$$
\Delta G=\Delta H-T \Delta S=\Delta E e l e+\Delta E V D W+\Delta G g b+\Delta G n p-T \Delta S
$$

Where $\Delta$ Eele and $\triangle E V D W$ are the total gas-phase energy/Molecular mechanics energy $(\Delta \mathrm{EMM}), \Delta \mathrm{Ggb}$ and $\Delta \mathrm{Gnp}$ refer to polar and non-polar solvation free energies respectively, and $\mathrm{T} \Delta \mathrm{S}$ is conformational entropy upon binding. The solute and the solvent dielectric constant values were set to 80 . The entropy was calculated as described in the methods reported by Wang et al. ${ }^{36}$ given in equation 2 and 3 ,

$$
\begin{gathered}
\text { Entropy }=S=\sum_{i=1}^{N} w i(S A S i+k * B S A S i \mid) \quad \ldots \text { (2) } \\
\text { Where } \mathbf{B S A S} \mathbf{i}=\mathbf{4} \boldsymbol{\pi}(\mathbf{r} \mathbf{i}+\mathbf{r} \mathbf{p r o b})^{\mathbf{2}}
\end{gathered}
$$

Here the $\mathrm{N}$ is the number of atoms in a molecule, wi, SASi, BSASi, ri denotes the weight, the solvent-accessible surface area, buried solvent accessible surface area, calculated using Equation 2 and the radius of the $\mathrm{i}$ atom respectively. The probe radius, rprob, was set to 0.08 $\mathrm{nm}$. The term $\mathrm{k}$ is the adjustable parameter with the values taken from the same work.

In their approach, the conformational entropy of a molecule, $\mathrm{S}$, can be obtained by summing up all atoms' contributions, no matter whether they are buried or exposed. The two types of surface areas SAS and BSAS, were weighted to estimate the contribution of an atom to $\mathrm{S}$. Atoms having the same atom type shared the same weight. A general parameter $\mathrm{k}$ is applied to balance the contributions of the two types of surface areas. Since we obtained the enthalpic and entropic term, it was possible to obtain the complexes' binding free energies. The inner product of the residues present on the interface in the complex enabled us to match if the same residues are involved in interface formation in two different complexes. The values range from 0 , which represents no match to 1 that represents the perfect match. Numerical value equal to or greater than 0.5 is usually considered a good match. In the present study, the inner product, $\gamma$, have been found between vectors Ac and A1, A2, and A3 respectively where,

$\mathbf{A c}=$ residue wise contribution to the interface area of the $\mathrm{M}^{\text {pro }}$ in $\mathrm{M}^{\text {pro }}-Z 4492329$ complex . $\mathbf{A 1}=$ residue wise contribution to the interface area of the $\mathrm{M}^{\text {pro }}$ in $\mathrm{M}^{\text {pro }}$-Ergotamine complex. $\mathbf{A 2}=$ residue wise contribution to the interface area of the $\mathrm{M}^{\text {pro }}$ in the $\mathrm{M}^{\text {pro }}$-Naldemedine complex.

A3 = residue wise contribution to the interface area of the $\mathrm{M}^{\text {pro }}$ in $\mathrm{M}^{\mathrm{pro}}$-Nilotinib complex.

The ith element of vector $A$ is the contribution of the ith residue of the protein to the interface of the complex. As a case study, a $\gamma$ value of 0.67 suggests a $67 \%$ match in the residues involved in the protein for two different complexes Ac and A1.

$$
\gamma=(\mathrm{Ac}) \overrightarrow{\mathrm{A}} \cdot(\mathrm{A1}) \overrightarrow{\mathrm{r}}=\mathbf{0 . 6 7}
$$


Table-2: Binding energy scores of the primary screened top-ranked inhibitors by Autodock Vina.

\begin{tabular}{|c|c|c|c|c|c|c|c|}
\hline S. No. & Compound Structure & Name (Formula/ ZINC ID) & $\begin{array}{c}\text { Docking } \\
\text { Score } \\
\left(\text { kcal.mol }^{-1}\right) \\
\end{array}$ & $\begin{array}{l}\text { Molecular Weight } \\
\text { (Daltons) }\end{array}$ & $\log P$ & $\begin{array}{c}\text { No. of H- } \\
\text { bond } \\
\text { Donors }\end{array}$ & $\begin{array}{l}\text { No. of H- } \\
\text { Bond } \\
\text { Acceptors }\end{array}$ \\
\hline 1. & (1) & $\begin{array}{c}\text { Ergotamine } \\
\mathrm{C}_{33} \mathrm{H}_{35} \mathrm{~N}_{5} \mathrm{O}_{5} / \text { ZINC52955754 }\end{array}$ & -9.5 & 581.673 & 1.991 & 3 & 6 \\
\hline 2. & & $\begin{array}{c}\text { Vorapaxar } \\
\mathrm{C}_{29} \mathrm{H}_{33} \mathrm{FN}_{2} \mathrm{O}_{4} / \text { ZINC3925861 }\end{array}$ & -9.3 & 492.591 & 5.63 & 1 & 5 \\
\hline 3. & & $\begin{array}{c}\text { Paliperidone } \\
\mathrm{C}_{23} \mathrm{H}_{27} \mathrm{FN}_{4} \mathrm{O}_{3} / \mathrm{ZINC} 4214700\end{array}$ & -9.2 & 426.492 & 3.081 & 2 & 6 \\
\hline 4. & & $\begin{array}{c}\text { Nilotinib } \\
\mathrm{C}_{28} \mathrm{H}_{22} \mathrm{~F}_{3} \mathrm{~N}_{7} \mathrm{O} / \text { ZINC6716957 }\end{array}$ & -9.0 & 529.526 & 6.356 & 2 & 7 \\
\hline 5. & & $\begin{array}{c}\text { Irinotecan } \\
\mathrm{C}_{33} \mathrm{H}_{38} \mathrm{~N}_{4} \mathrm{O}_{6} / \text { ZINC1612996 }\end{array}$ & -9.0 & 586.689 & 4.091 & 2 & 8 \\
\hline 6. & & $\begin{array}{c}\text { Naldemedine } \\
\mathrm{C}_{32} \mathrm{H}_{34} \mathrm{~N}_{4} \mathrm{O}_{6} / \mathrm{ZINC} 100378061\end{array}$ & -8.9 & 570.646 & 3.48 & 4 & 8 \\
\hline 7. & & $\begin{array}{c}\text { Eltrombopag } \\
\mathrm{C}_{25} \mathrm{H}_{22} \mathrm{~N}_{4} \mathrm{O}_{4} / \text { ZINC11679756 }\end{array}$ & -8.9 & 442.475 & 4.565 & 0 & 7 \\
\hline 8. & & $\begin{array}{c}\text { Dihydro-ergotamine } \\
\mathrm{C}_{33} \mathrm{H}_{37} \mathrm{~N}_{5} \mathrm{O}_{5} / \text { ZINC3978005 }\end{array}$ & -8.8 & 583.689 & 2.081 & 4 & 5 \\
\hline 9. & 20 & $\begin{array}{c}\text { Noxafil } \\
\mathrm{C}_{37} \mathrm{H}_{42} \mathrm{~F}_{2} \mathrm{~N}_{8} \mathrm{O}_{4} / \mathrm{ZINC} 28639340\end{array}$ & -8.7 & 700.791 & 4.573 & 1 & 12 \\
\hline
\end{tabular}




\section{Results and discussion}

\section{1- Structure-based virtual screening results}

FDA approved drug molecules were screened primarily based on their calculated binding energy score during docking studies. Three FDA approved drug molecules, viz- Ergotamine, Nilotinib, and Naldemedine were selected based on their binding affinities towards $\mathbf{M}^{\text {pro }}$ active site. Among all docked drug molecules, the highest binding energy score was of Ergotamine, which was $-9.5 \mathrm{kcal}^{\mathrm{mol}}{ }^{-1}$. The molecule is a $\alpha-1$ adrenergic agonist vasoconstrictor, used for treating chronic migraine disorders. The molecule interacted within $\mathrm{M}^{\text {pro }}$ active site, forming conventional carbon-hydrogen bonds with residues Glu166, His41, and Asn142. A $\pi$-Sulfur bond was evident with Met165 along with four alkyl and $\pi$-alkyl interactions with Met49 and Pro168 residues. A $\pi-\pi$ T-shaped interaction was visualized with His41 residue, and several van der Waals interactions were evident in the docked complex (Figures-3B and 4B).

The next two drug molecules interacted with $\mathrm{M}^{\text {pro }}$ of SARS-CoV2 were Nilotinib and Naldemedine having predicted binding energy score of $-9.0 \mathrm{kcal}^{\mathrm{mol}}{ }^{-1}$ and $-8.9 \mathrm{kcal}^{\mathrm{mol}}{ }^{-1}$. Nilotinib is a tyrosine kinase inhibitor used in the second-line treatment of Chronic Myelogenous Leukaemia (CML). Unlike Ergotamine, the Nilotinib molecule in the docked complex has shown quite a different molecular interactions with SARS-CoV2 $\mathrm{f}$ active site residues. There were two conventional hydrogen bonds between Nilotinib and $\mathrm{M}^{\text {pro }}$ within active site involving Glu166 and Asn119 residues. The docked assembly consisted of three halogen bonds in between Nilotinib molecules and amino acid residues Leu141, His163, and Met 165. The three alkyl and $\pi$-alkyl bonds with Cys 145 and His163 and multiple van der Waals interactions were evident in the docked assembly of $\mathrm{M}^{\text {pro }}$ with Nilotinib molecule (Figures-3C and 4C). An unfavourable but peculiar donor-donor interaction with Glu143 was seen in the docked molecule. The $\mu$-opioid receptor antagonist-Naldemedine, which is used to prevent opioid-induced constipation in adult patients, formed four in total (conventional and carbonhydrogen) hydrogen bonds with residues Thr26, Asn119 and Gly143. There were three alkyl and $\pi$-alkyl interactions found with the residues His41 and Met49. A $\pi$ - $\pi$ stacked interaction with Tyr118 and many van der Waals interactions were also evident in the docked assembly (Figures-3D and 4D).

In contrast to the top three screened FDA drugs, the native hydrolase inhibitor (Z44592329), PDB ID-5R83, was docked on the enzyme's active site. The binding energy was $-6.0 \mathrm{kcal}^{-\mathrm{mol}^{-}}$ ${ }^{1}$, much lower than the screened FDA approved inhibitors. The inhibitor formed five hydrogen bonds, including the conventional and non-conventional carbon-hydrogen bonds in total with the residues Phe140, His 163, Met165, and Glu166. The inhibitor formed two alkyl and $\pi$-alkyl bonds with Met49 and Met165 residues while interacting with His41, His141, and Met165 through $\pi$ - $\pi$ T-shaped interactions. Few van der Waals interactions were also evident in the docked structure (Figures-3A and 4A).

More van der Waals interactions were visible in drug docked structure than native hydrolase inhibitor docked assembly, which might be the reason for the higher binding energy score of drug molecules. Considering the substantial difference in the binding energy score and 
significant interactions between drug molecules and $\mathrm{M}^{\text {pro }}$ active site, it seems possible for these FDA approved molecules to act as $\mathbf{M}^{\text {pro }}$ inhibitors and abolish its enzymatic activity.

\section{2- Molecular Dynamic Simulation Analysis}

The stability of the drug molecule in the active site serves as an accurate quantifier to assess the fitness of potential inhibitors. $\mathbf{M}^{\text {pro }}$ complex with all drug molecules and the native hydrolase inhibitor Z44592329 at the active site were subsequently subjected to molecular dynamic simulation. The protein backbone stability was assessed in terms of root-mean-square deviation (RMSD) values of the protein-drug complexes. The RMSD values were calculated concerning the initial frame of protein-drug complex and plotted, as shown in figure 5A. The RMSD values of $\mathrm{M}^{\text {pro }}$ and $\mathrm{M}^{\text {pro }}$-ligand complexes increased gradually from 0 to $5 \mathrm{~ns}$ and remained stable after throughout the trajectory. The stability for residues was assessed in terms of root-mean-square fluctuation (RMSF) values of protein $\mathrm{C} \alpha$ backbone averaging over the 8595ns interval, as shown in figure 5B. The RMSF values for the active site residues in $\mathrm{M}^{\text {pro }}$ drug complexes ranged less than $0.7 \AA$; however, no significant change in RMSF values was observed as compared to that of free $\mathrm{M}^{\text {pro }}$ except the $\mathrm{N}$-terminal and $\mathrm{C}$-terminal regions, which showed higher flexibility. Also, the mean inner product value was at least 0.5 or more for all the $\mathrm{M}^{\text {pro }}$-drug complexes indicating a good match for the residues involved in the $\mathrm{M}^{\text {pro }}$ Z44592329 complex, as shown in figure 6A.

Table-3. Free binding energy $\left(\Delta G^{\circ}\right)$ of the top-ranked putative inhibitors against Mpro of SARS-CoV-2.

\begin{tabular}{|c|c|c|c|c|}
\hline Drug & $\begin{array}{c}\Delta H \\
\left.\text { (kcal.mol }^{-1}\right) \\
\end{array}$ & $\begin{array}{c}T \Delta S \\
\left(\mathrm{kcal} . \mathrm{mol}^{-1}\right)\end{array}$ & $\begin{array}{c}\Delta G \\
\left(\mathrm{kcal} . m o l^{-1}\right)\end{array}$ & $\begin{array}{c}\mathrm{pKi} \\
(\mu \mathrm{M})\end{array}$ \\
\hline $\begin{array}{c}\text { Ergotamine } \\
(\mathbf{5 2 9 5 5 7 5 4 )}\end{array}$ & $-33.962 \pm 2.068$ & $-24.102 \pm 0.024$ & $-9.86 \pm 2.092$ & 6.974 \\
\hline $\begin{array}{l}\text { Naldemedine } \\
\text { (100378061) }\end{array}$ & $-29.382 \pm 3.594$ & $-21.149 \pm 0.027$ & $-8.233 \pm 3.621$ & 5.823 \\
\hline $\begin{array}{l}\text { Nilotinib } \\
(6716957)\end{array}$ & $-22.499 \pm 3.958$ & $-23.974 \pm 0.061$ & $1.471 \pm 4.019$ & 1.036 \\
\hline $\begin{array}{c}\text { Crystal Ligand } \\
\text { (Z44592329) }\end{array}$ & $-19.348 \pm 1.904$ & $-11.409 \pm 0.019$ & $-7.939 \pm 1.923$ & N.D ${ }^{*}$ \\
\hline
\end{tabular}

\section{3- Binding Free-energy Calculation}

The meta-stable region for all the complex, i.e., 85-95ns of the molecular dynamics simulation, was selected to calculate the binding free energy, as mentioned in the table- 3 . When compared with native inhibitor (Z44592329), Ergotamine and Naldemedine showed lower Gibbs free energy, which was in accordance as per the docking score obtained by PyRx. Nilotinib showed higher Gibbs free energy, which could be explained due to the lower conformational entropy 
with some fluctuations. The conformational entropy for Ergotamine, Naldemedine, and Z44592329 was constant, as shown in figure 6B. Since MM-PBSA is a computational method based on approximations and shows an intermediate level of accuracy between empirical scoring, and alchemical perturbation methods, there will always be a scope of improvement in the calculated values. The $\mathrm{p} K_{\mathrm{i}}$ (the negative logarithm of predicted inhibition constant) of the screened drug molecules, Ergotamine, Nilotinib, and Naldemedine, was 6.974, -1.036 and 5.823 micromolar $(\mu \mathrm{M})$ respectively as calculated during the meta-stable region of the simulation (Table-3).

\section{Conclusions}

SARS-CoV2 $\mathrm{M}^{\text {pro }}$ regulates the replication and transcription of the virus through the generation of non-structural proteins (NSPs) from viral polyprotein, which ultimately forms a replicasetranscriptase assembly. Hence, SARS-CoV2 $\mathrm{M}^{\text {pro }}$ is an attractive drug target for structure-based drug design and development. Blocking of the $\mathrm{M}^{\text {pro }}$ enzyme active site of SARS CoV2 will have prophylactic effects on viral infection and progression. Structure-based screening through docking of FDA approved drugs in the active site of SARS-CoV2 $\mathrm{M}^{\text {pro }}$ helped to identify three potential inhibitors of main protease activity; viz- Ergotamine, Nilotinib, and Naldemedine. MD trajectory analysis and calculated binding energy (MMPBSA) verifies the stability of these drugs in the active site of the enzyme. The obtained result strengthens the idea- the binding of these drugs with SARS-CoV2 $\mathrm{M}^{\text {pro }}$ could impede the essential proteolytic function of the enzyme causing inhibition of viral replication and disease progression. However, the identified drug molecules need to be tested through in vitro and clinical experiments for their efficacy before being utilized in prophylactic therapy and management of SARS-CoV2 infection.

\section{Acknowledgment}

The authors acknowledge the High Performance Computing (HPC) facility at the Indian Institute of Technology Delhi (IIT Delhi), New Delhi, to provide computational resources for conducting the research.

\section{Funding}

Corresponding Author (GS) is a Senior Research Fellow (SRF) under the doctoral fellowship scheme of the Department of Biotechnology (DBT), Government of India, New Delhi. No Grants or funding was received to conduct the published research work.

\section{Declaration/ Competing Interests}

We declare that the authors do not have any competing interests associated with the published manuscript.

\section{Availability of data and materials}

The datasets used and analysed during the published work are available from the corresponding author on reasonable request. 


\section{Author's contribution}

GS, TKC, GG, and VS conceived and designed this study. GS, VS, and RS conducted the computational analysis. GS, VS, RS, analysed the raw data. GG and TKC interpreted the raw data and provided critical comments. All the authors assisted in drafting, reviewing, and approved the final version of the manuscript.

\section{Abbreviations}

3CL ${ }^{\text {pro: }}$ Chymotrypsin Like Protease, ACE-2: Angiotensin-Converting Enzyme-II, BSAS: Buried Solvent Accessible Surface area, CML: Chronic Myelogenous Leukaemia, CoV: Coronavirus, COVID-19: Coronavirus Disease, MERS-CoV: Middle East Respiratory Syndrome Coronavirus, $\mathbf{M}^{\text {pro: }}$ Main Protease, PDB: Protein Data Bank, RMSD: Root Mean Square Deviation, RMSF: Root Means Square Fluctuation, SARS-CoV: Severe Acute Respiratory Syndrome Coronavirus, SARS-CoV2: Severe Acute Respiratory Syndrome Coronavirus-2, SAS: Solvent Accessible Surface area, WHO: World Health Organization.

\section{References:}

1. WHO. WHO Timeline. World Health Organization https://www.who.int/newsroom/detail/27-04-2020-who-timeline---covid-19 (2020).

2. Zhong, N. S. et al. Epidemiology and cause of severe acute respiratory syndrome (SARS) in Guangdong, People's Republic of China, in February, 2003. Lancet (2003) doi:10.1016/S0140-6736(03)14630-2.

3. Wang, N., Shang, J., Jiang, S. \& Du, L. Subunit Vaccines Against Emerging Pathogenic Human Coronaviruses. Frontiers in Microbiology (2020) doi:10.3389/fmicb.2020.00298.

4. Du, L. et al. The spike protein of SARS-CoV - A target for vaccine and therapeutic development. Nature Reviews Microbiology (2009) doi:10.1038/nrmicro2090.

5. WHO. World Health Organization COVID-19 Dashboard. World Health Organization COVID-19 Dashboard https://covid19.who.int/ (2020).

6. Li, Q. et al. Early transmission dynamics in Wuhan, China, of novel coronavirusinfected pneumonia. New England Journal of Medicine (2020) doi:10.1056/NEJMoa2001316.

7. Chen, N. et al. Epidemiological and clinical characteristics of 99 cases of 2019 novel coronavirus pneumonia in Wuhan, China: a descriptive study. Lancet (2020) doi:10.1016/S0140-6736(20)30211-7.

8. Li, F. Structure, Function, and Evolution of Coronavirus Spike Proteins. Annu. Rev. Virol. (2016) doi:10.1146/annurev-virology-110615-042301.

9. Huang, C. et al. Clinical features of patients infected with 2019 novel coronavirus in Wuhan, China. Lancet (2020) doi:10.1016/S0140-6736(20)30183-5.

10. Guan, W. et al. Clinical characteristics of coronavirus disease 2019 in China. N. Engl. 
J. Med. (2020) doi:10.1056/NEJMoa2002032.

11. He, F., Deng, Y. \& Li, W. Coronavirus disease 2019: What we know? Journal of Medical Virology (2020) doi:10.1002/jmv.25766.

12. Su, S. et al. Epidemiology, Genetic Recombination, and Pathogenesis of Coronaviruses. Trends in Microbiology (2016) doi:10.1016/j.tim.2016.03.003.

13. Zhou, F. et al. Clinical course and risk factors for mortality of adult inpatients with COVID-19 in Wuhan, China: a retrospective cohort study. Lancet (2020) doi:10.1016/S0140-6736(20)30566-3.

14. Andersen, K. G., Rambaut, A., Lipkin, W. I., Holmes, E. C. \& Garry, R. F. The proximal origin of SARS-CoV-2. Nature Medicine (2020) doi:10.1038/s41591-0200820-9.

15. Pawar, A. Y. Combating Devastating COVID -19 by Drug Repurposing. Int. J. Antimicrob. Agents (2020) doi:10.1016/j.ijantimicag.2020.105984.

16. Sarma, P. et al. In-silico homology assisted identification of inhibitor of RNA binding against 2019-nCoV N-protein (N terminal domain). J. Biomol. Struct. Dyn. (2020) doi:10.1080/07391102.2020.1753580.

17. Zhou, Y. et al. Network-based drug repurposing for novel coronavirus 2019nCoV/SARS-CoV-2. Cell Discov. (2020) doi:10.1038/s41421-020-0153-3.

18. Hoffmann, M. et al. SARS-CoV-2 Cell Entry Depends on ACE2 and TMPRSS2 and Is Blocked by a Clinically Proven Protease Inhibitor. Cell (2020) doi:10.1016/j.cell.2020.02.052.

19. Wrapp, D. et al. Cryo-EM structure of the 2019-nCoV spike in the prefusion conformation. Science (80-. ). (2020) doi:10.1126/science.aax0902.

20. Anand, K. et al. Structure of coronavirus main proteinase reveals combination of a chymotrypsin fold with an extra $\alpha$-helical domain. EMBO J. (2002) doi:10.1093/emboj/cdf327.

21. Lei, J., Kusov, Y. \& Hilgenfeld, R. Nsp3 of coronaviruses: Structures and functions of a large multi-domain protein. Antiviral Res. 149, 58-74 (2018).

22. Zhang, L. et al. Crystal structure of SARS-CoV-2 main protease provides a basis for design of improved a-ketoamide inhibitors. Science (2020)

doi:10.1126/science.abb3405.

23. Yang, H. et al. The crystal structures of severe acute respiratory syndrome virus main protease and its complex with an inhibitor. Proc. Natl. Acad. Sci. U. S. A. (2003) doi:10.1073/pnas.1835675100.

24. Khan, R. J. et al. Targeting SARS-CoV-2: a systematic drug repurposing approach to identify promising inhibitors against 3C-like proteinase and 2'-O-ribose methyltransferase. J. Biomol. Struct. Dyn. (2020) doi:10.1080/07391102.2020.1753577.

25. Arun, K. G., Sharanya, C. S., Abhithaj, J., Francis, D. \& Sadasivan, C. Drug repurposing against SARS-CoV-2 using E-pharmacophore based virtual screening, molecular docking and molecular dynamics with main protease as the target. $J$. 
Biomol. Struct. Dyn. 0, 1-12 (2020).

26. Naik, V. R. et al. Remdesivir (GS-5734) as a therapeutic option of 2019-nCOV main protease - in silico approach. J. Biomol. Struct. Dyn. 0, 1-14 (2020).

27. Shamsi, A. et al. Glecaprevir and Maraviroc are high-affinity inhibitors of SARSCoV-2 main protease: possible implication in COVID-19 therapy. Biosci. Rep. (2020) doi:10.1042/BSR20201256.

28. Trott, O. \& Olson, A. J. AutoDock Vina: Improving the speed and accuracy of docking with a new scoring function, efficient optimization, and multithreading. J. Comput. Chem. (2009) doi:10.1002/jcc.21334.

29. Guan, B., Zhang, C. \& Zhao, Y. HIGA: A running history information guided genetic algorithm for protein-ligand docking. Molecules (2017) doi:10.3390/molecules22122233.

30. Kagami, L. P. et al. LiGRO: a graphical user interface for protein-ligand molecular dynamics. J. Mol. Model. (2017) doi:10.1007/s00894-017-3475-9.

31. Abraham, M. J. et al. Gromacs: High performance molecular simulations through multi-level parallelism from laptops to supercomputers. SoftwareX (2015) doi:10.1016/j.softx.2015.06.001.

32. Sousa Da Silva, A. W. \& Vranken, W. F. ACPYPE - AnteChamber PY thon Parser interfacE. BMC Res. Notes (2012) doi:10.1186/1756-0500-5-367.

33. Humphrey, W., Dalke, A. \& Schulten, K. VMD: Visual molecular dynamics. J. Mol. Graph. (1996) doi:10.1016/0263-7855(96)00018-5.

34. Pettersen Ef Fau - Goddard, T. D. et al. UCSF Chimera--a visualization system for exploratory research and analysis. J. Comput. Chem. (2004).

35. Kumari, R., Kumar, R. \& Lynn, A. G-mmpbsa -A GROMACS tool for highthroughput MM-PBSA calculations. J. Chem. Inf. Model. (2014) doi:10.1021/ci500020m.

36. Wang, J. \& Hou, T. Develop and test a solvent accessible surface area-based model in conformational entropy calculations. J. Chem. Inf. Model. (2012)

doi:10.1021/ci300064d. 


\section{Figures}

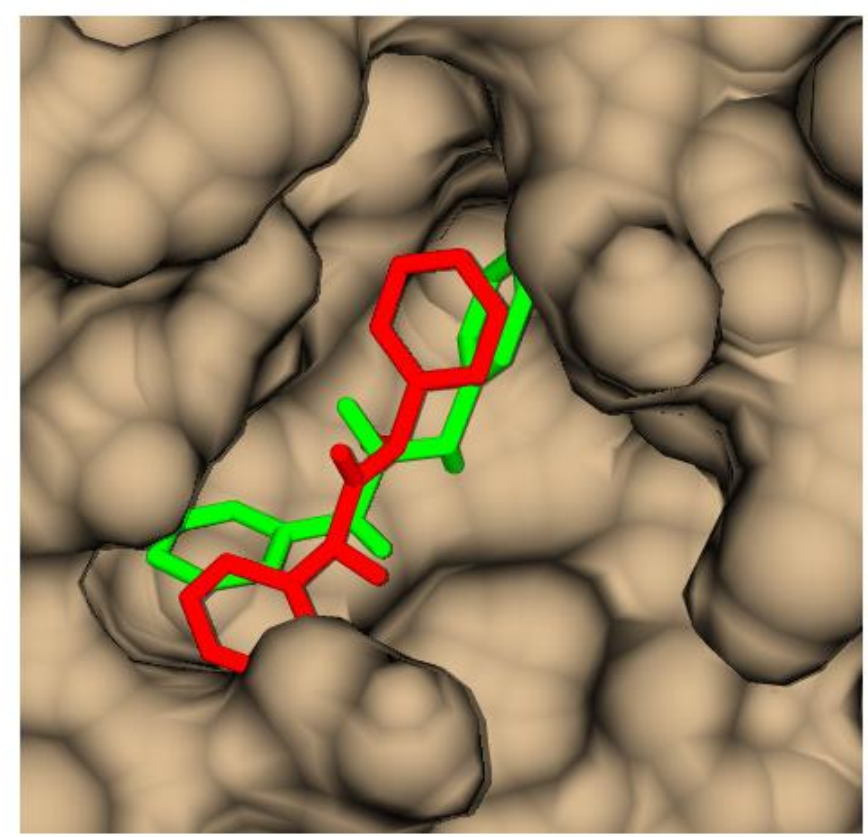

Figure 1. Overlapped representation of Crystal (Green) and Docked (Red) pose of native hydrolase inhibitor (Z44592329) at the active site of Mpro.
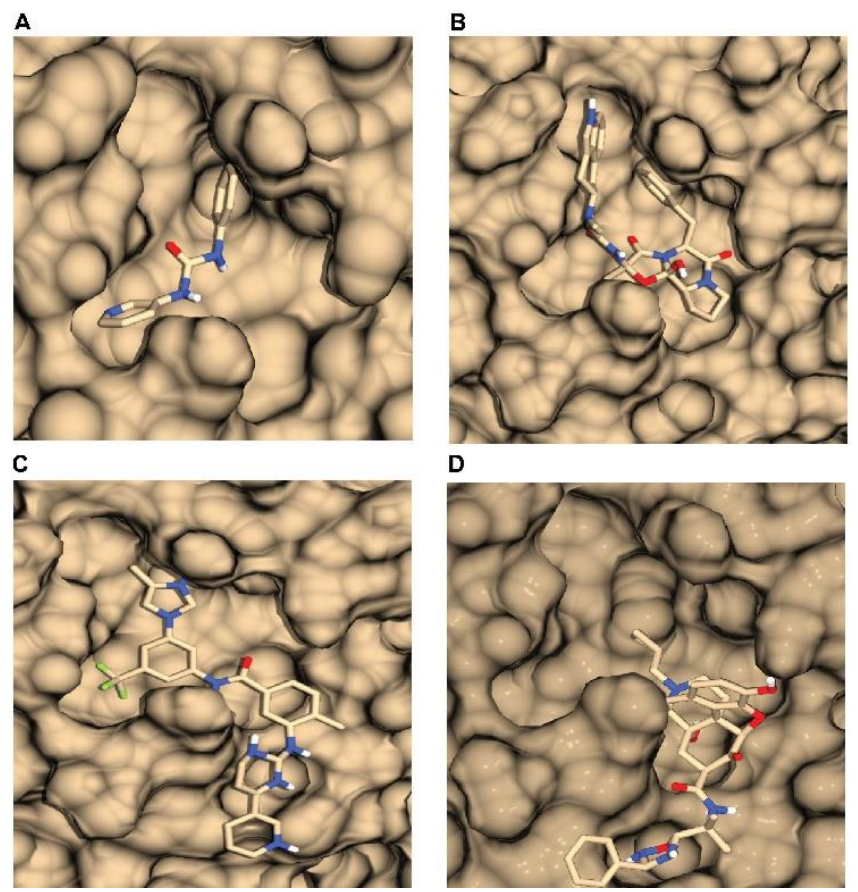

Figure 2. Docked Surface view representation of the top-ranked compounds. Mpro complexes: (A) Z44592329, (B) Ergotamine, (C) Nilotinib and (D) Naldemedine. 

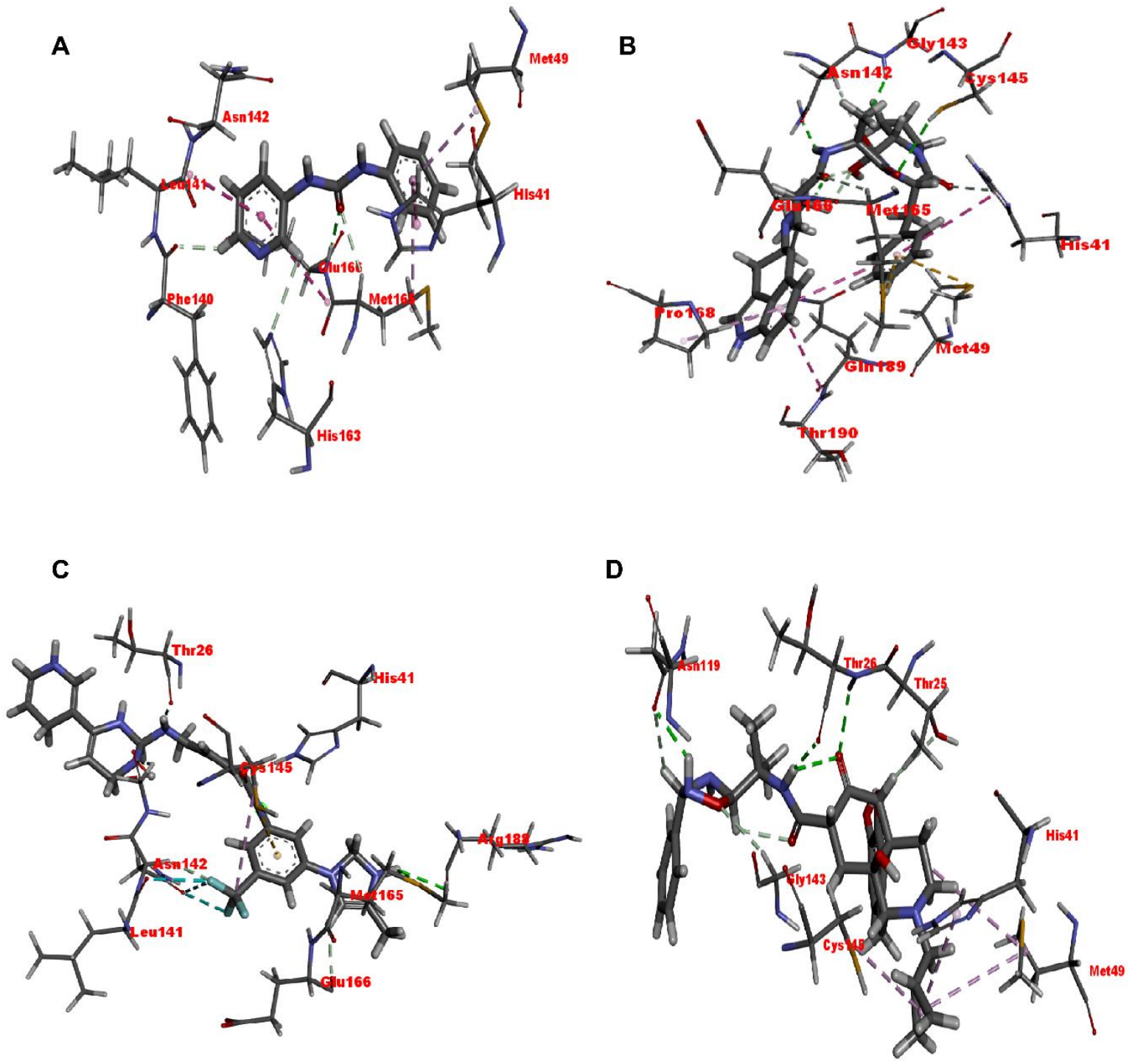

Figure 3. 3D-interaction illustration of top-ranked compounds. Mpro complexes: (A) Z44592329, (B) Ergotamine, (C) Nilotinib and (D) Naldemedine. 

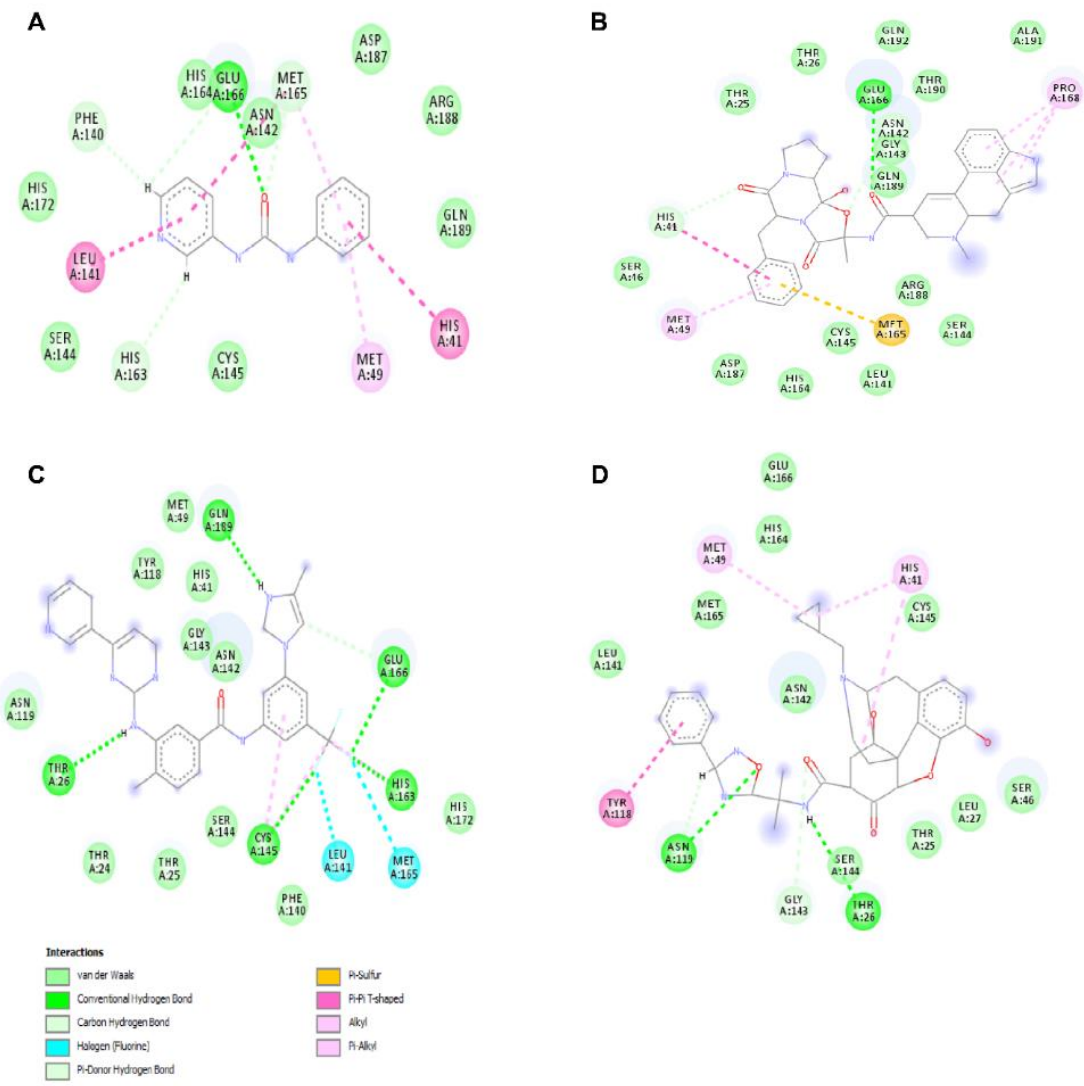

Figure 4. 2D-interaction illustration of top-ranked compounds. Mpro complexes: (A) Z44592329, (B) Ergotamine, (C) Nilotinib and (D) Naldemedine.
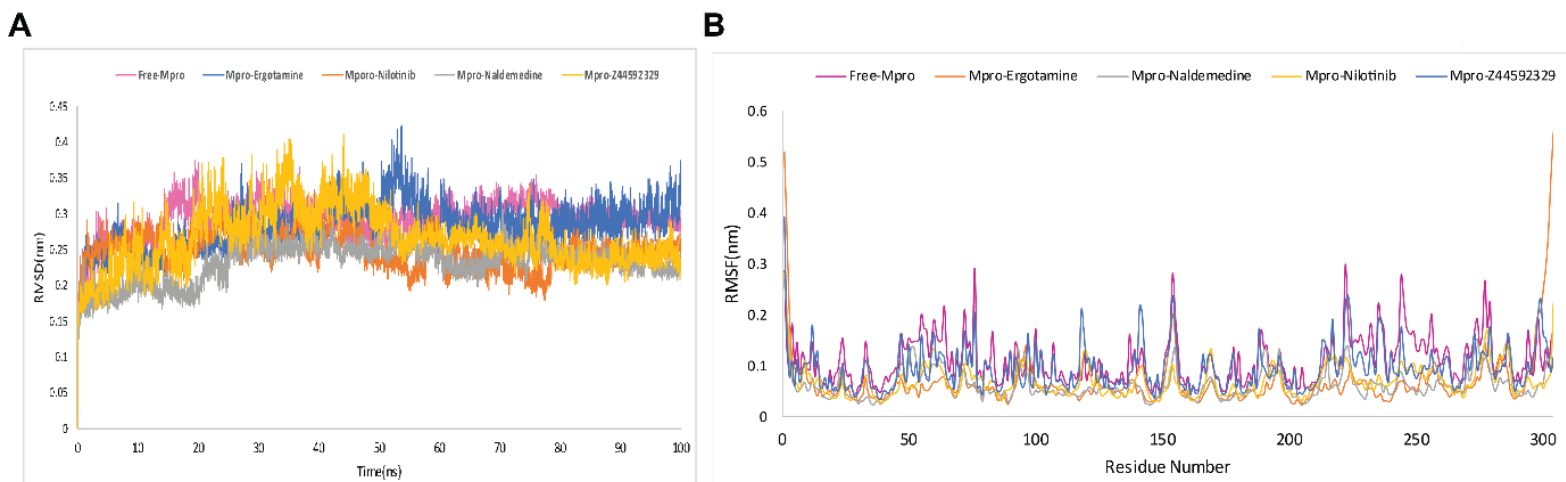

Figure 5. Molecular dynamic (MD) simulation of $\mathrm{M}^{\text {pro }}$-ligand molecule complex, (A) RMSD plots of free and drug complexed Mpro and (B) RMSF plots of free and drug complexed Mpro. 
A

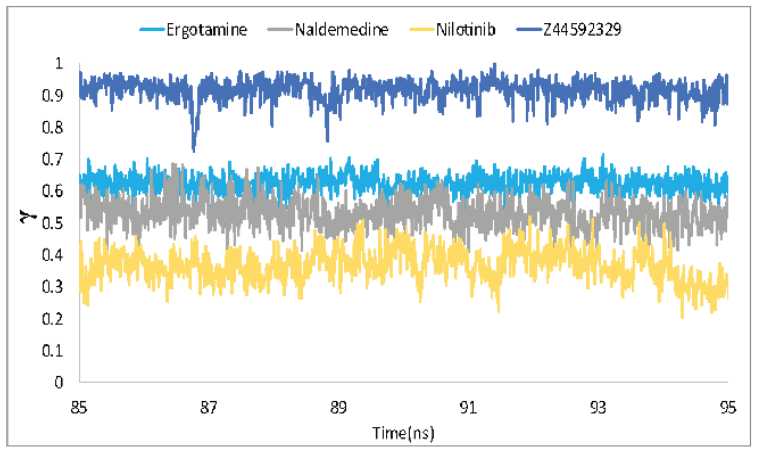

B

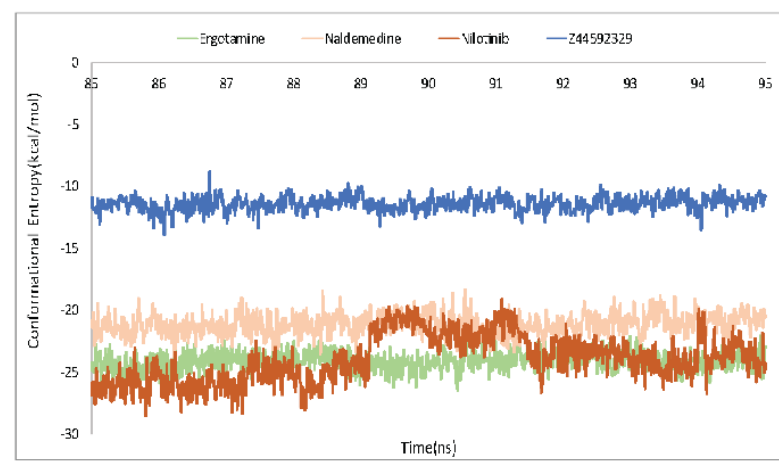

Figure 6. (A) Inner-product calculation between Mpro-Z44592329 and Mpro-screened drugs complex and (B) Conformational entropy of Mpro-screened drugs. 